\title{
'I have a story to tell': Researching migrant women's experiences of female genital mutilation and gender-based violence in Ireland and Europe
}

\section{Siobán O’Brien Green ${ }^{1}$}

\begin{abstract}
This article presents insights and practical lessons learned from multiple studies the author has undertaken and participated in as principal or co-researcher and/or provided expert guidance to in Ireland and Europe. These studies primarily focus on gender-based violence (GBV) and female genital mutilation (FGM) and given their foci, have an implicit need for cognisance of child protection, legislation and onward referral procedures. The research issues of interest are often considered taboo, private, not to be discussed outside immediate family and shameful. There are multiple practical and logistical barriers, as well as language and psycho-social obstacles, to participating in, and undertaking, research on these issues. The article discusses the approaches and routes taken to recruit women affected and impacted by the issues of FGM and GBV for research studies. The responsibility on researchers to present research study findings in a sensitive manner which does not add stigma to marginalised and vulnerable groups, but that enables policy makers to utilise the research for legislative and practical purposes, is also discussed.
\end{abstract}

Keywords: gender-based violence (GBV); female genital mutilation (FGM); migration; ethics; stigma; research design

\section{Ussher Postgraduate Fellow/PhD Candidate}

Address for correspondence: Trinity Research in Childhood Centre, Trinity College Dublin, Dublin 2, Ireland. obriengs@tcd.ie

Date of first (online) publication: 2nd October 2018 


\section{Introduction}

As society in Ireland has changed in relation to increasing numbers of nationalities and ethnicities over the past number of years and mobility within an ever-expanding European Union (EU) occurs, social scientists have a responsibility to ensure that their research is both reflective of the issues raised by population changes and responsive to their implications (McGinnity, et al., 2018). This ongoing reassessment of research design and approaches is particularly relevant in relation to gender-based violence (GBV) in its multiple forms of presentations and lived experiences.

Fontes and McCloskey (2011, p.151) state, 'Violence against women does not look the same across cultures,' therefore, research on GBV (with an inclusive scope to fully explore and collect forms of experiences and presentations) is needed to accurately capture this multicultural reality. As populations transform and shift in Europe, an additional challenge is how to research and understand complex social issues such as GBV within altering populations and societies to ensure that data is representative and robust, and that recommendations and policy measures arising address the needs of the whole population. The current Irish National Action Plan on Domestic, Sexual and Gender-based Violence acknowledges that communities of particular vulnerability, including migrants, require specific responses which are evidence based (Cosc, 2016). Despite the knowledge that population changes require both an inclusive and targeted research approach to reflect shifts in potential study samples and the range of issues being researched, a further challenge is often the hidden nature of the issue within communities of interest and the broader population.

Gender-based violence (GBV) is defined as violence that disproportionately affects persons of a particular gender, and it includes all forms of violence against women, including domestic or intimate partner violence, sexual violence, and female genital mutilation (FGM)(European Institute for Gender Equality, 2015b). According to recent research, the global lifetime prevalence of physical and/or sexual intimate partner violence amongst all ever-partnered women was 30\% (World Health Organization, 2013). A survey commissioned by the European Union Agency for Fundamental Rights (FRA) (2014), consisting of face-to-face interviews with 42,000 women across the $28 \mathrm{EU}$ member states, established that one in three had experienced some form of physical and/or sexual assault since the age of 15 a lifetime prevalence similar to the WHO research. The FRA survey also noted a $43 \%$ lifetime prevalence of psychological violence by a current or ex-partner. These figures are based on nationally representative population samples from the $28 \mathrm{EU}$ countries, but in depth statistically significant analysis based on a woman's ethnicity and migration history is not possible utilising the FRA survey data (Goodey, 2017). As a result, additional research and targeted sampling is required to gain a more comprehensive understanding of the GBV experiences of migrant women living in the EU. Types and manifestations of GBV that may have been once observed or 
known to happen 'elsewhere' are increasingly emerging as issues for women living in Ireland and Europe (Women's Health Council, 2009).

Female genital mutilation (FGM), as defined by the World Health Organization, is the partial or total removal of the external female genitalia, or any practice that purposely alters or injures the female genital organs for non-medical reasons. FGM has serious impacts on women's health, bodily integrity and the potential for safe birth. It is a grave form of GBV and recognised as a human rights violation (O'Brien Green, 2014). The United Nations High Commissioner for Refugees (2013) state that approximately 20,000 women and girls from FGM practicing counties seek asylum in the EU on an annual basis. Van Baelen et al. (2016) estimate that, based on the Europe-wide 2011 censuses, there are 576,068 women and girls living in the EU, Switzerland and Norway who have undergone FGM. This figure is considered to be an underestimation, and it does not include girls living in Europe who have not undergone FGM but who may be at risk of FGM. Irish figures from this study indicate that approximately 5,277 women and girls aged ten and over have undergone FGM and were living in Ireland in 2011 - a figure that has potentially increased since then (ibid, 2016). Although the figures for women living with FGM in Europe and Ireland are not insubstantial, they obscure the diversity of the ethnicities, countries of origin/birth, and other variables and differences in the women who make up the numbers reported.

If research is to inform the development of policy and legislative responses, as well as evidence-based practice, service delivery and resource allocation, it must aim to be representative of the whole of society and bring voices and experiences that may initially appear peripheral to be heard and acknowledged. This article will draw on the author's experience in conducting and advising research studies investigating GBV and FGM, with a key focus on ethnic minority and/or migrant women in Ireland and the EU. Drawing on experience of research study design, sampling and methods used, conducting focus groups, surveys and interviews, the challenges and learning from researching hard to reach populations will be shared as a basis of a model for informing work with similar populations.

\section{Why is this research population hard to reach?}

There are multiple practical and logistical barriers, as well as linguistic and psychosocial obstacles, to participating in and undertaking research on the areas of GBV and FGM in migrant populations. Violence is recognised as a challenging and sensitive topic to research (Fraga, 2016). The mainly female participants in such studies can be marginalised and hidden, either waiting for legal/resident status to be granted or working in informal sectors and subject to discrimination (Montoya and Rolandsen Agustín, 2013). They may have never disclosed to others their personal 
experiences of GBV, FGM, sexual violence, etc., and they may have experienced multiple forms of GBV prior to, during, and after migration, including trafficking for the purpose of exploitation (Women's Health Council, 2009). They may not speak the official language(s) of the country in which they are living, and much of their lives and movements may be managed by male family members and/or partners/ husbands (Women's Health Council, 2009). The issues outlined in this article, GBV and FGM, are often considered taboo, private, not to be discussed outside the immediate family, and shameful (Mergaert, et al., 2015). These research areas intersect with a woman's sexual and reproductive health, pregnancy and birth, integration and isolation, marriage and immediate family, and cultural traditions, all leading to research challenges, including initial recruitment and retention of interviewees (Mergaert, et al., 2015).

The World Health Organization (WHO) (2016) suggests that the sensitive nature of researching GBV requires specific considerations in relation to ethics, safety and sampling. These challenges in undertaking research - given the immense personal threats and risks that women have had, or with which they are currently living highlight the need for robust research design, to ensure the safety of the research participants and, in some cases, the researcher. Therefore, the topic of GBV requires that considered and appropriate safety measures be an integral part of the study design, data collection and data storage, to ensure ongoing participant safety, even once the study has been completed (Jaquier, et al., 2011). How women are engaged and accessed to participate in research, heard and listened to by researchers, and have their histories documented, as well as where the research articles and reports are published, places much responsibility on the researcher and the research funder, in relation to safety, confidentiality, re-victimisation and vicarious trauma. The ethical considerations in relation to researching domestic violence are also intricate and have entailed much discussion. The biomedical model of vulnerable research populations may not be entirely suited to social science research on the topic of violence against women, and robust and well-defined models are scarce or may need to be adapted to local/regional needs and circumstances (Downes, et al., 2014).

The population of interest - migrant women - can be highly mobile, or even transitory, and living with anxiety related to pending/probable deportation and returning to their country of origin at any point in time; the potential for longitudinal studies or multiple data collection episodes with the desired study sample can be reduced. Therefore, a sensitive, well-thought-out approach is required when designing these research studies. Often, attempts to research these topics - given their hidden nature, complexity and sensitivity - have relied on proxy interviews with service providers, community spokespersons, or officials in roles pertinent to violence against women, FGM or child protection (Leye, et al., 2014). As a result, the populations most impacted can be 'spoken for', and effectively silenced via research. Some research studies have attempted to overcome this issue by purposeful sampling for supplemental samples or specific study focus groups 
with vulnerable or marginalised sub-populations within the national population to attempt to capture their experiences (McBride, et al., 2012; McGee, et al., 2002; Watson and Parsons, 2005).

As a result of the difficulty in recruiting some populations, the utilisation of spokespersons or key community leaders occurs in place of direct research with the sample. This approach can illuminate understanding about an issue, but it should not replace attempts to speak with those who have personal experience of the topic. Over-reliance on community leaders or spokespeople can potentially mute or silence others from participating in research and cannot fully reflect the lived experiences of the issues being researched. However, where it is not appropriate or safe to do so, or perhaps due to time and resource pressures from research funders, alternatives to direct data collection with the population of interest may be required.

\section{Methodological challenges for carrying out research on GBV and FGM with migrant women}

\section{Research study design}

'In the case of gender-based violence research, the risks are potentially large, but so too are the risks of ignorance, silence and inaction' (Ellsberg and Heise, 2005, p.45).

Bearing in mind the challenges associated with researching GBV and accessing the study population, the research study design will vary, given the time frame, study budget, team size and composition, and the desired, commissioned or required outputs. Pilot testing consent forms, interview guides and questionnaires, and ensuring that study materials have been translated into the appropriate language(s) and/or conform to plain English or other accessibility requirements is resource intensive and time consuming. This step can be overlooked, and then, during fieldwork, the study documents are either too complex or not in the language required, acting as a deterrent to study recruitment and participation, or not allowing for fully informed consent to be obtained. To maximise the readability of all the study documents, the research studies the author has participated in and/or conducted were informed by learning from a Plain English writing course (Plain English Campaign, 2018) and the WHO Informed Consent Form Template for Qualitative Studies (World Health Organization, 2018). This was to ensure that potential study interviewees who did not speak English as a first language or who had literacy issues were still able to understand the studies, relevant study documentation and what their participation entailed.

Building trust with key contacts, such as communities, services that may assist with recruitment, or women as potential participants takes time. Meeting in 
person or talking on the phone prior to data collection can reassure these contacts of the robustness of the study design in relation to participant safety and ethical requirements, the researcher's credentials and the sensitivity of their approach, and that there will be outputs from the study that have the possibility of making changes for other women in similar situations in the future. This cannot be guaranteed in all cases, so a strong track record in terms of transitioning research into policy, practice, legislation and service resourcing may need to be demonstrated in order to encourage participation in research studies and gatekeeper cooperation. Adequate budgets and realistic time frames for study recruitment are recommended, with enough flexibility to respond to emerging issues/challenges and to mitigate risks. Trust and subsequent disclosure to the interviewer may be facilitated or inhibited by additional factors such as sex of the researcher (Fraga, 2016). Women may prefer to be interviewed by male or female interviewers and this should be ascertained prior to interviewing (Zimmerman and Watts, 2003). Lastly, envisaging the study design in such a way that research findings will be useful to key professionals interacting with migrant women such as social workers, social care workers, health and medical staff, immigration and border officials, police, etc., to inform and leverage amelioration and long-term change, is vital.

\section{Research participant and study sample recruitment and retention}

Determining accurate sample sizes when little is known about the study population in question, and if mobility is a feature of the population, is exceptionally challenging. While national census data may represent an indicator of a population, varying migrant flows for numerous push-and-pull factors can mean that the data is not fully up to date and may not be an accurate indicator of the study population (European Institute for Gender Equality, 2013). For those who are undocumented or wish to remain 'under the radar' of government departments and agencies, it becomes even more challenging to begin to assess a sub-population size and ensure that adequate samples are calculated within a given population, to provide meaningful and valid research results. In addition, certain groups in the population may not appear in census figures, such as prisoners, undocumented migrants, and asylum seekers, therefore distorting actual population numbers and lessening confidence in sample size calculations (Leye, et al., 2014).

For some sensitive research topics, and if the sample population of interest is small, new methods to estimate prevalence of GBV are required. A statistical extrapolation model utilising census and other relevant available data on migrant women and girls was used to estimate the number of women who have undergone FGM and are were living in Ireland in 2008, 2010 and 2013 (Leye, et al., 2014). The 'extrapolation-of-FGM-prevalence-countries-data-method' does not use face to face data collection methods, instead it relies on global FGM rates extrapolated 
into national census and/or other relevant population figures (Bansal, et al., 2013). Although utilised in many EU countries to give an indication of the female population who have undergone FGM this method has several limitations. These limitations include a lack of ethnicity data in census data and a lack of disaggregation based on other factors relevant to FGM risk (European Institute for Gender Equality, 2013). However, in the absence of other methods, and given the sensitivity of the issue being researched, this method allows for preliminary estimates of women living in the EU who have undergone FGM (mostly prior to migrating to the EU). It also allows for appropriate legislation, health care, support and prevention interventions to be developed and resourced based on needs of the estimated population and it provides clear, replicable methodology which can be repeated over time to examine trends (Van Baelen, et al., 2016).

The worries and concerns about disclosing and sharing very intimate and hard-todiscuss issues such as GBV can be an immediate deterrent to research participation. Avoiding participant attrition and generating snowball samples, which utilise peer networks to reach into groups unfamiliar with taking part in research studies and who may have legitimate fears and concerns that mitigate against (full) participation in these studies, is a further concern. Accessing participants to take part in research is a key challenge in relation to GBV research, and more so when migrant and minority ethnic women are the core sample. Utilising peer and community networks to drive interviewee recruitment and generate snowball samples may take more time than initially anticipated.

Gatekeepers from relevant services and communities can be utilised to access the study population and promote participation in the study. This may lead to power and control dynamics being exerted and a subsequent sample selection bias. Gatekeepers are unlikely to select poor attendees/engagers or clients who might be critical of the services offered or managed by the gatekeepers, therefore, the voices of the most marginalised are unlikely to be heard in research, as they are unlikely to be asked to participate. There may be efforts to present services positively, so only certain clients are informed about the research by the gatekeepers (Vearey, et al., 2017). Acknowledging that there will be both a level of self-selection by participants and an additional layer of participant recruitment by gatekeepers needs to be clear in the research data reporting and sample explanation. A frank and transparent statement of the study sample recruitment strategy, any challenges encountered, the methods used to communicate with potential study participants and gatekeeper(s), and shortcomings in relation to the findings can help clarify any doubts or questions that readers may have.

An over-reliance on strict study inclusion criteria in relation to nationalities/ ethnicities may not reflect the lived realities of research participants, and there is a need to be flexible. Given that migration routes into Europe are often long and convoluted, via multiple countries and involving periods of time in different regions, in addition to intermarriages and parenting between mixed ethnicity 
and/or nationality partners, a simple selection of nationality/ethnicity is often not reflective of the reality of female migrants' lives (United Nations High Commissioner for Refugees, 2013). This emerged as an issue with focus groups when exploring the effects of migration on continuation/rejection of FGM in a European diaspora setting. One woman expressed interest in participating in the focus groups, but she was not from one of the key countries of origin with a documented prevalence of FGM. When the researcher called her to inform her that she did not meet the study inclusion criteria, she responded that she was very eager to attend the focus group, stating, 'I have a story to tell'. She participated in the first of three focus groups, had experience of FGM risk through a former partner, who was from an FGM-practising country, and was crucial in terms of snowball recruitment for two of the subsequent focus groups (Mergaert, et al., 2015). The valuable and insightful contribution on the topic being researched and the additional participants whom she brought to the groups would have been lost if strict and inflexible study inclusion criteria had been meticulously followed.

\section{Compensation and childcare}

Compensation for study participants and/or interviewees can be an issue of concern for research ethics committees and research study budgets. While compensation can be seen as incentivising potential interviewees to participate by means of pressure and, therefore, biased study findings, it can also mitigate against interviewees' travel and childcare expenses and lost earnings when giving their time to participate in a study. Globally, women earn less than men, they may have experienced financial hardship as a result of leaving a violent relationship, and they may have been victims of economic abuse (European Institute for Gender Equality, 2014; Watson and Parsons, 2005). Recognition of these facts and support for the expenses incurred to participate in research studies should be part of every GBV research study design. To truly value the time that women take to travel, arrange childcare, and not work in order to attend interviews, there is a strong argument for adequate compensation upon completing a study interview/survey/focus group.

Sullivan and Cain (2004) address the issue of providing adequate compensation to women participating in research interviews. They state:

'Compensation for participating in any research should be high enough to show respect for women's time and expertise, but not so high that it might coerce women into participating when they would rather not' (ibid, 2004, p. 615).

Meaningful, realistic and user-friendly incentives, rewards or expenses for research participation should be offered to interviewees, the income levels of hardto-reach groups may be lower than that of the general population, and for some 
individuals taking part in the research means that they are not earning money (Kenny, et al., 2014). Their access to transport, childcare and phone or email to communicate with the researcher and to attend interviews or focus groups can also be limited.

User preference should guide the type of reward/thank-you item for research participants, ensuring that it is not hard to use and can be done so anonymously and conveniently. The acceptability of cash to immediately cover participants' transportation, childcare and communication expenses (confirming attendance at an interview or seeking directions to the location of a focus group, for example) should not be underestimated (Sullivan and Cain, 2004). Unfortunately, for some research ethics committees, there is a mistrust of cash, as it could be utilised to coerce participation in research studies, whereas, in many cases, it simply allows for partaking in what could be a prohibitively costly activity. A uniform compensative sum for all research participants, regardless of the number of children requiring childcare or the method of transport used to attend the focus group, reduces pressure on researchers to 'assess' the individual amount of compensation required. It allows for the transparent promotion of participation in the research, with a clearly set compensative amount, and it reduces time needed at the end of the interview or focus group to distribute compensation to participants..

For migrant women, a lack of extended family and strong community networks may mean that childcare supports are absent, and the only way that women can participate in research is with their babies or infants. This can present challenges for the researcher, as time will need to be allocated for baby feeds and breaks, and noise from the child can be an issue for the transcription intelligibility of interviews. Planning for the childcare needs of research participants can mitigate this in some ways (O'Brien Green, 2017). Children should not be present in interviews or focus groups, if they are verbal, for any research related to GBV. This is for two principal reasons: the content can be upsetting to the child, and there exists a possibility of children being later quizzed (for example, by the perpetrator of GBV) as to what their mother has told others, potentially exacerbating risks and compromising participant safety (Zimmerman and Watts, 2003).

\section{During the data collection}

Prior to all interviews, the researcher needs to read through the informed consent form with the interviewee, check her understanding of the text, and respond to any clarifications that may be required. Reminders during the research process, interview or focus group about consent, and not to share more than that with which they are comfortable, may need to be noted as prompts in the interview guide/ questionnaire. As focus groups may require comfort or food breaks, reminders of the consent agreement as the regroup occurs can ensure lucidity on the issue. The use of 'research coercion', in which the researcher tries to extract the maximum amount 
of data in a set time frame, potentially beyond what the participant is comfortable with sharing or discussing, needs to be avoided. The possibility for the interviewee to decline to answer certain questions or to retract her consent throughout the data collection should be clearly provided (Fontes, 2004).

Although interview and focus group guides may have predetermined parameters on what will be discussed, being open - a willingness to really listen to the issues and topics that participants wish to, and feel comfortable enough to, articulate and share - requires experienced and sensitive researchers. Through this openness, unanticipated issues can arise in the data collection process, but they are issues of great relevance to the participants. For example, the impact of having undergone FGM on sexual relations in marriage was not envisaged as a key issue in focus groups in the European Institute for Gender Equality FGM and risk study (Mergaert, et al., 2015). However, it emerged in the first focus group as a subject that caused distress and concern, and it was implicated in marriage breakdown. Although the topic was very sensitive and could be considered private, women felt that it impacted on their communities in relation to marriage failure, and that it was a negative outcome of FGM that was rarely discussed and acknowledged. As a result, referral routes to services and support needed by participants to cope with this unforeseen topic needed to be established quickly (Mergaert, et al., 2015). Voodoo and witchcraft can also emerge in interviews in relation to domestic violence, as acts and methods used by perpetrators. Respectful responses and reassurances to alleviate participant fears and concerns, and potential shock from the researcher, are needed.

\section{Responding to participant and researcher distress}

Researching and asking women about their experiences of GBV can be distressing. Women are often presented as victims in their lives, not only in the legal sense, imposing a role and label that women may not associate with their lived experiences and understanding of GBV and, in particular, FGM. Women may not, until an interview or focus group, associate any of their negative physical or mental health repercussions with the ritual of FGM. Indeed, they may not recall undergoing FGM, and they can become distressed when the experience is described by others in a focus group, and the subsequent harms and impacts are outlined (Mergaert, et al., 2015).

Participant protocols are required in advance of starting data collection and may include responses to the following:

- a disclosure of child abuse;

- a disclosure of intent to harm self, including suicide;

- a disclosure of intent to harm another person;

- the need for specialised counselling or supports in relation to GBV, domestic violence and/or FGM; and 
- guidelines and procedures for managing distress during and after the interview/ focus group.

The protocols devised and used should be mindful of national mandatory/legal reporting and disclosure requirements, especially in the case of an ongoing risk of child abuse. They should include appropriate referral routes and contact details for services, including out-of-hours/weekend services, to which to refer interviewees, and the costs, if any, associated with these services. Lists of dedicated services and information sources in the areas of health, police, GBV, immigration, legal aid and child protection should be available to researchers in case they are needed. Protocols should be mindful of institutional and funder ethical research guidelines and requirements.

In research on GBV and FGM the reality of researching an issue that can be traumatic and distressing for the researcher or research team during interviews, focus groups and/or during the transcription of interview recordings emerges. As a result, supports for researchers to avoid burnout, resultant poor data collection and analysis, and researcher attrition (due to distress with the topics and other issues related to vicarious trauma) need to be built into the study design and researcher management and support (World Health Organization, 2016). Vicarious trauma and distress for the researcher is an often overlooked issue when researching complex and distressing issues with participants who are exceptionally stigmatised and marginalised. Building in support, debriefing, and other processes into the research design, as well as opportunities to discuss the study challenges, is crucial to retaining researcher reflexivity and completing the study (Medical Research Council, 2015). Supervision and additional researcher supports may be required, in some cases.

\section{Managing expectations and power and privilege differentials}

Halse and Honey (2005) emphasise 'hearing the voices and silences smothered by the conversations of others' in relation to moral dilemmas that social scientists face when researching challenging topics ethically and bringing the experiences of women into the public domain. The act of telling another person about violence experienced can be transformative, in terms of naming and identifying the issue and accessing a range of supports (peer, professional, police, etc.) and by being believed by another person. Literature suggests that participation in qualitative research studies on domestic violence enables victims to use their experiences to help others. Women may find the interview a cathartic, meaningful experience and feel valued and listened to as they recount their own personal story (Downes, et al., 2014).

While the interviewee may, in best-case research scenarios, feel respected, listened to, and that her experience is acknowledged as a crime or abuse of human and/or children's rights, the power of a social scientist/researcher to effect immediate change 
on the circumstances that enabled the violence to occur is limited. When women who are experiencing, or have experienced, multiple challenges - for example, those relating to their legal status, family reunification, work permits, etc., in addition to potentially multiple forms of GBV - it is imperative that researchers are realistic as to how participating in a research study may, or may not, (eventually) inform and/ or change policy and practice. In this regard, researchers need to be able to discuss their previous achievements in utilising their research to inform and shape policy, service provision, and, in some cases, legal change with potential participants. They should also be ready to address and manage any participant expectations that sharing the details of their life experiences will affect rapid change in the current circumstances of the participants. Despite the expertise, contacts and potential relative power and privilege differentials between the researcher and participants, it may be months before the research is in the public domain and has any possibility of impacting or instigating change. However, researchers can be organised and prepared to refer interviewees to additional services and facilities relevant to the topics being researched and support them to access the appropriate services or assistance in relation to their concerns (O'Brien Green, 2017).

It is also important to acknowledge that while the main topic of interest to the researcher may be GBV or FGM, the current concerns or experiences that interviewees or focus group participants wish to disclose and discuss may be completely different or contemporaneous. As a result, contradictions between the stated research study aims and the immediate and pressing needs and issues of research participants may emerge. This can be the result of a recent experience, events in the media, or, indeed, events happening to a participant's extended family or community in her country or region of origin, about which she feels distressed and concerned. A participant may wish to have their experiences heard by the focus group and researcher for a range of reasons: to validate them, to have a listening ear, to vocalise and process what has happened to them, and to share, make sense of, or seek answers to what has occurred in their life. We, as researchers, may try to pigeonhole the 'story' or recollections to fit into our research question or study design, rather than providing a space to respect the research participant and the contribution that they wish to make. Although effective and confident group facilitation is important in guiding the overall discussion, it can be a challenge to 'quiet' or 'contain' a participant who feels that their issues need to be disclosed and shared. Conscious and ongoing reminding of the topics for discussion and limits to the confidentiality that can be guaranteed by the researcher may need to be restated during the research process, as well as time provided post-interview to articulate and share other issues and concerns with the researcher.

At the end of the survey, interview or focus group, the researcher can leave, both physically and psychologically, the topics or issues being investigated. This option may not be available to the participant, due to the long-term effects and impacts of GBV, especially FGM, her current living circumstances, such as living in direct 
provision (a hostel style communal accommodation for asylum seekers in Ireland) or a women's refuge, and/or ongoing contact with, or living in proximity to, her abuser (Montoya and Rolandsen Agustín, 2013; Women's Health Council, 2009). Researchers need to be sensitised to this reality and acknowledge that the options and choices that they have, or can exercise, at the end of the research process may not be the same as those of their participants. This may be upsetting for the participant, as the circumstances that have led to her current living situation have been outlined to the researcher, and there may be little at this point in time that the latter can do to amend or change them. The researcher can ask, at the end of the interview, about the long-term visions and goals that the interviewees have for their lives and families, while being aware of the fact that their current circumstances are not ideal, that they are temporary, and will potentially change. Emphasising a woman's strengths and terminating interviews with a positive tone is recommended, and it can signify the benefit of participating in research as an empowering process, and not necessarily an extractive one (Ellsberg and Heise, 2005).

\section{After the data collection}

When working with marginalised individuals, the researcher is responsible for providing information, as well as collecting it. This is an important aspect of ethical research (Zimmerman and Watts, 2003). Researchers should be prepared for followup contact from participants in relation to issues that emerged during the research process or could have been re-stimulated by recalling events or issues from the past. Even though referral routes and information on support services and contacts should have been provided to all participants post-focus group or interview, they may feel more comfortable contacting the researcher, as a trusting connection may have been established. The researcher may be the most knowledgeable person whom the research participant has encountered to date on issues relevant to the research topic, including legislation, welfare, immigration status, GBV services, etc. As a result, she may contact the researcher post-interview to seek advice, referrals, and further information related to the topic or her situation. The researcher should be ready to respond and support onward referrals.

Following on from hearing others share their experiences, or once a level of trust has been established with a researcher, participants may wish to share more information, or they may recall more details that they feel are relevant to the research and of interest to the researcher. Sharing contact details and creating a space for this to happen can allow for more and richer data to be collected, but reminding participants of the limitations of confidentiality and informed consent principles is necessary, especially if this sharing occurs via email or phone, and not within a face-to-face interview setting. Ensuring adherence to ethical approval received for the research, or seeking an amendment to the ethical approval, may be required to 
adapt to data collection via unanticipated routes or contexts.

Information packs for participants to take away, read through and engage with after the research should be prepared prior to data collection. All the packs should contain uniform contents, such as referral routes and information on relevant services and contact details. This is to avoid any embarrassment, especially within focus groups, for a participant having to select which information that they want or need. Pre-prepared information packs can be distributed to all participants at the end of the group (O'Brien Green, 2017). The information provided should be clear enough to allow for ease of self-referrals to services and include the details of any fees or costs associated with accessing the services. Information may need to be provided in a range of languages, and appropriate to the research participants' literacy levels. Including the thank-you and compensation for the participants' time with the information is another method of ensuring that it is disseminated effectively. The information packs should be easy to conceal, discreet and portable, and should only be taken by the research participant if it is safe to do so and will not put her at increased risk after her interview (Jaquier, et al., 2011).

\section{Publication and presentation of research study findings}

When we discuss any particular kind of violence against women with a particular culture, we risk contributing to overgeneralization and stereotypes (Fontes and McCloskey, 2011, p.151).'

The responsibility on researchers to present study findings in a sensitive manner that does not add stigma to marginalised groups, but that enables policymakers to utilise the research for legislative and practical purposes is crucial. The sensitivity of research findings for vulnerable populations can add to stigma, further marginalisation and fuel negative stereotypes (European Institute for Gender Equality, 2013). How and when research findings are presented needs to be carefully considered, so as to maximise the potential for amelioration of the complex social issue(s) being studied for the population in question, and in order to minimise the utilisation of research results for harmful, potentially sensational or out-of-context presentation of the findings. Utilising the findings emerging from research in academic, policy and legislative settings and relevant professional sectors such as social work, child protection, domestic violence and policing to promote understanding, more sensitive responses, and well-resourced specialist services needs to be planned for. Researchers can also involve participants (where safe and appropriate to do so) in disseminating the research findings and results, and attending and participating in research launches to fully acknowledge their involvement and thank them for their essential contributions. The findings or reports may need to be emailed or posted to study participants for dissemination, where possible, in order to share the results 
with them if it is not feasible or safe for them to attend research launches or seminars.

In addition, research findings must be completely non-identifiable, not only for ethical and confidentiality requirements of research conduct, but, in some cases, because there might be ongoing legal processes, such as refugee status determination, right-to-reside visa applications, and legal proceedings resulting from crimes such as violence, sexual assault and FGM. Identifiable details in the public domain could be utilised by perpetrators or within legal settings. This may require, as Fontes suggests, a greater-than-usual use of anonymisation of all details and changing some key aspects of participant demographics to maintain the safety and confidentiality of those participating in the research while still being true to the overall thrust of their experiences (Fontes, 2004). As domestic violence is a patterned, repetitive and ongoing crime, it is likely that the impacts, repercussions or risks for research participants will change, escalate and de-escalate over time. While it might be considered safe and appropriate for a person to be interviewed or participate in a focus group at one point in time, her circumstances can be altered considerably due to issues such as perpetrator mobility, decisions on migration status or the right to remain in a country, and/or changes to her health/financial circumstances. The future impact of a publication of research findings needs to be considered by researchers while being mindful of changes that can make their research cohort both more or less vulnerable over time.

\section{Conclusions}

This article has outlined and addressed some of the technical and practical issues and challenges of undertaking research on GBV, including FGM, with migrant women in Ireland and the EU, based on the author's own research experiences. Participation in conscientiously designed research can be part of a process for the women of re-examining issues, such as GBV and FGM, in the context of their new country. Reaching and recruiting migrant women, often through gatekeepers, given their mobility and marginalisation is a crucial challenge which can be overcome, again with thoughtful study design, sufficient time and resources and comprehensive planning. However, there exists a danger of over-researching a particular population or topic of interest. There is no potential value in continually probing and exploring issues, such as FGM and GBV, with groups and individuals without creating subsequent change and impact as a result of research findings and outputs generated.

The Council of Europe Convention on preventing and combating violence against women and domestic violence (referred to as the Istanbul Convention) obliges states that have ratified it in relation to actions on research on all forms of GBV. States are required under the Istanbul Convention to support research and conduct population-based surveys at regular intervals to assess the prevalence levels of GBV 
and ensure that research undertaken is available to the public (European Institute for Gender Equality, 2015b). As a result research in Ireland, and in other Council of Europe member states, on GBV and FGM is likely to increase as more countries ratify the Istanbul Convention. Ensuring that this future research approaches the task with an understanding of the differing needs, experiences and safety issues for potential research participants amongst European populations is imperative (Holt, et al., 2017). This is in order to build a body of evidence that reflects and captures all the stories that women are willing to share and tell us, as social scientists and researchers, and to ensure we utilise our research to make women's lives safer in the future.

\section{Acknowledgments}

Thanks from the author to Professor Trevor Spratt, Trinity College Dublin (TCD), Lucy Deegan Leirião, Greg Sheaf (TCD) and Dr Jo Murphy Lawless (TCD).

\section{References}

Bansal, S., Breckwoldt, M., O’Brien Green, S. and Mbugua, S. (2013) Female genital mutilation: Information for health-care professionals working in Ireland. 2nd ed. Dublin: AkiDwA, Health Service Executive and Royal College of Surgeons in Ireland

Cosc (2016) Action Plan: Second National Strategy on Domestic, Sexual and Gender Based Violence 2016-2021. Dublin: Government Publications Office

Downes, J., Kelly, L. and Westmarland, N. (2014) Ethics in Violence and Abuse Research -a Positive Empowerment Approach. Sociological Research Online, 19, 1, 1-13

Ellsberg, M. and Heise, L. (2005) Researching Violence Against Women: A Practical Guide for Researchers and Activists. Washington DC: World Health Organization

European Institute for Gender Equality (2013) Female Genital Mutilation in the European Union and Croatia. Vilnius: European Institute for Gender Equality

European Institute for Gender Equality (2014) Estimating the costs of gender-based violence in the European Union. Vilnius: European Institute for Gender Equality

European Institute for Gender Equality (2015b) Strategic framework on violence against women 2015-18. Vilnius: European Institute for Gender Equality

European Union Agency for Fundamental Rights (2014) Violence Against Women: an EU-wide Survey. Luxembourg: Publications Office of the European Union

Fontes, L. A. (2004) Ethics in Violence Against Women Research: The Sensitive, the Dangerous, and the Overlooked. Ethics \& Behavior, 14, 2, 141-174

Fontes, L. A. and McCloskey, K. A. (2011) Cultural issues in violence against women. In C. 
M. Renzetti, J. L. Edleson and R. Kennedy Bergen (Eds.) Sourcebook on Violence Against Women. Thousand Oaks, CA: Sage (pp. 151-171)

Fraga, S. (2016) Methodological and ethical challenges in violence research. Porto Biomedical Journal, 1, 2, 77-80

Goodey, J. (2017) Violence Against Women: Placing Evidence From a European Union-Wide Survey in a Policy Context. Journal of Interpersonal Violence, 32, 12, 1760-1791

Halse, C. and Honey, A. (2005) Unravelling Ethics: Illuminating the Moral Dilemmas of Research Ethics. Signs, New Feminist Approaches to Social Science, 30, 4, 2141-2162

Holt, S., Devaney, J. and Överlien, C. (2017) Concluding Remarks. In S. Holt, C. Överlien and J. Devaney (Eds.) Responding to Domestic Violence: Emerging challenges for policy, practice and research in Europe. London: Jessica Kingsley (pp. 341-347)

Jaquier, V., Johnson, H. and Fisher, B. S. (2011) Research Methods, Measures and Ethics. In C. M. Renzetti, J. L. Edleson and R. Kennedy Bergen (Eds) Sourcebook on Violence Against Women. California: SAGE (pp. 23-48)

Kenny, N., NíRian, A. and Daly, M. (2014) Domestic Violence: A Guide for General Practice. Dublin: Quality in Practice Committee, Irish College of General Practitioners

Leye, E., Mergaert, L., Arnaut, C. and O'Brien Green, S. (2014) Towards a better estimation of prevalence of female genital mutilation in the European Union: interpreting existing evidence in all EU Member States. Genus, 70, 1, 99-121

Mergaert, L., Arnaut,C., Exterkate, M., O'Brien Green, S., Strid, S. and Leye, E. (2015) Estimation of girls at risk of female genital mutilation in the European Union. Vilnius: European Institute for Gender Equality

McBride, O., Morgan, K. and McGee, H. (2012) Irish Contraception and Crisis Pregnancy Study 2010 (ICCP-2010): A Survey of the General Population. Dublin: HSE Crisis Pregnancy Programme

McGee, H., Garavan, R., de Barra, M., Byrne, J. and Conroy, R. (2002) The SAVI report: sexual abuse and violence in Ireland a national study of Irish experiences, beliefs and attitudes concerning sexual violence. Dublin: The Liffey Press and Dublin Rape Crisis Centre

McGinnity, F., Grotti, R., Russell, H. and Fahey, É. (2018) Attitudes to Diversity in Ireland. Dublin: Irish Human Rights and Equality Commission and the Economic and Social Research Institute

Medical Research Council (2015) Guidelines for the prevention and management of vicarious trauma among researchers of sexual and intimate partner violence. Pretoria: Medical Research Council

Montoya, C. and Rolandsen Agustín, L. (2013) The Othering of Domestic Violence: The EU and Cultural Framings of Violence against Women. Social Politics: International Studies in Gender, State \& Society, 20, 4, 534-557

O'Brien Green, S. (2014). What is FGM? Association for Improvements in the Maternity Services Journal, 26, 2, 14-15

O'Brien Green, S. (2017) Organising focus groups: Process and logistics. In E. Leye and G. Coene (Eds.) Researching female genital mutilation/cutting. Brussels: Brussels University Press (pp. 85-90) 
Plain English Campaign (2018) Plain English Course [Accessed on 29 May 2018 at http:// www.plainenglish.co.uk/services/training/in-house-courses-available/plain-english-inhouse-course.html]

Sullivan, C. and Cain, D. (2004) Ethical and Safety Considerations When Obtaining Information From or About Battered Women for Research Purposes. Journal of Interpersonal Violence, 19, 5, 603-618

United Nations High Commissioner for Refugees (2013) Too much pain: female genital mutilation $\&$ asylum in the European Union a statistical overview. Brussels: United Nations High Commissioner for Refugees

Van Baelen, L., Ortensi, L. and Leye, E. (2016) Estimates of first generation women and girls with female genital mutilation in the European Union, Norway and Switzerland. The European Journal of Contraception \& Reproductive Health Care, 21, 6, 474-482

Vearey, J., Barter, C., Hynes, P. and McGinn, T. (2017) Research ethics in practice: lessons from studies exploring interpersonal violence in different contexts. Families, Relationships and Societies, 6, 2, 273-289

Watson, D. and Parsons, S. (2005) Domestic Abuse of Women and Men in Ireland: Report of the National Study of Domestic Abuse. Dublin: National Crime Council and Economic and Social Research Institute

Women's Health Council (2009) Translating Pain into Action: A Study of Gender-Based Violence and Minority Ethnic Women in Ireland. Full Report. Dublin: Women's Health Council

World Health Organization (2013) Global and regional estimates of violence against women: prevalence and health effects of intimate partner violence and non-partner sexual violence. Geneva: World Health Organization

World Health Organization (2016) Ethical and safety recommendations for intervention research on violence against women. Building on lessons from the WHO publication Putting women first: ethical and safety recommendations for research on domestic violence against women. Geneva: World Health Organization

World Health Organization (2018) Informed Consent Form Template for Qualitative Studies. Geneva: World Health Organization. Accessed on 30 May 2018 at http://www.who.int/ rpc/research_ethics/informed_consent/en/

Zimmerman, C. and Watts, C. (2003) WHO ethical and safety recommendations for interviewing trafficked women. Geneva: World Health Organization [Accessed on 30 May 2018 at http:// www.who.int/rpc/research_ethics/informed_consent/en/] 